\title{
La gestión de la información como contenido curricular en la formación docente. Caso de una universidad cubana
}

\section{The Information Management as Curricular Content in Teachers' Formation. Case of a Cuban University}

\author{
Esperanza Asencio Cabot* \\ Universidad Central "Marta Abreu" de Las Villas. Cuba. \\ ORCID: https://orcid.org/0000-0002-7086-5240 \\ Nilda Ibarra López \\ Universidad Central "Marta Abreu" de Las Villas. Cuba. \\ ORCID: https://orcid.org/0000-0003-3830-1282
}

Received 05-12-20 Revised 07-25-20 Accepted 09-04-20 On line 09-29-20

*Correspondence

Email: easencio@uclv.cu
Cite as:

Asencio, E., \& Ibarra, N. (2020). La gestión de la información como contenido curricular en la formación docente. Caso de una universidad cubana. Propósitos y Representaciones, 8 (SPE2), e729. Doi: http://dx.doi.org/10.20511/pyr2020.v8nSPE2.729 


\section{Resumen}

El trabajo aborda la experiencia desarrollada en la Universidad Central "Marta Abreu" de Las Villas, Cuba, relacionada con la concepción e implementación de una propuesta curricular de programa optativo acerca de la actualización de la gestión de la información de los estudiantes en formación docente, para solucionar las carencias que en ese sentido presentaban los mismos. Se insiste en la importancia actual del tema tratado y se analiza el diseño metodológico de la experiencia, el proceso de determinación de necesidades, la caracterización del programa y la implementación de actividades docentes; dichas actividades se fueron construyendo desde la práctica, a partir del empleo de métodos de investigación, en especial el método de investigación-acción, por lo que estuvieron sometidas a ciclos de mejoramiento continuo durante todo el proceso vivido. Se presentan los resultados obtenidos y se destaca la medición de los indicadores a partir del desempeño de los estudiantes, considerando también los datos cualitativos recogidos durante el proceso. En general, se demostró que los estudiantes elevaron su nivel de dominio en los conocimientos, habilidades y valores relacionados con los contenidos abordados, así como su motivación por continuar elevando su cultura informacional para responder a las exigencias de del desarrollo científico técnico.

Palabras clave: Gestión de la información; cultura informacional; programa curricular optativo; formación docente; desarrollo científico técnico.

\section{Summary}

The paper deals the experience developed in the Central University "Marta Abreu" of the Villas, Cuba, related to the conception and implementation of a curricular proposal of optative program about the update of the information management of the students in teachers' formation, to solve the deficiencies that in that sense they presented. It is insisted on the present importance of the treated subject and it is analyzed the methodological design of the experience, the process of determination of necessities, the characterization of the program and the implementation of educational activities; these activities went constructing from the practice, from the use of research methods, in special the method of research-action, reason why they were put under cycles of continuous improvement throughout the lived process. The obtained results and the measurement of the indicators from the performance of the students are presented, also considering the gathered qualitative data during the process. In general, it was demonstrated that the students elevated their level of dominion in the knowledge, abilities and values related to the contents, as well as its motivation to continue elevating its informational culture to respond to the exigencies of the technical scientific development.

Keywords: Information management; informational culture; optative curricular program; teachers' formation; technical scientific development.

\section{Introducción}

El desarrollo científico-tecnológico que caracteriza la sociedad actual promueve el uso de la información y de las tecnologías para el cumplimiento con éxito de las diferentes actividades, por ello se requiere elevar de manera continua la cultura informacional de los profesionales en las diferentes áreas del saber. En el área educativa, resulta esencial asumir la tarea de comprender y difundir la cultura informacional a partir de la creación de ambientes de aprendizaje basados en los métodos actuales de trabajo con las tecnologías, con la información y en redes colaborativas (Uribe, 2013).

En los ámbitos de la educación superior y de la investigación, la constante introducción de innovaciones tecnológicas así como los acelerados flujos de la información, exigen a estudiantes y profesores no sólo un alto nivel de conocimientos en el campo de las tecnologías de información y comunicación, sino además una permanente renovación de las capacidades para 
orientarse en el ciberespacio, usar la información creativamente y participar en redes colaborativas de aprendizaje (Uribe, 2013; Aleixandre, Alcaide, González, \& Alonso, 2011; Varela, 2007).

La universidad debe responder a las nuevas expectativas como el aprendizaje a lo largo de la vida, de ahí que la gestión de la información y el desarrollo de habilidades informacionales resultan esenciales para desarrollar la propia educación de las personas (Winston, Barbosa, Marciales, \& Castañeda, 2010; Pinto, et al., 2009) y constituyen la piedra angular para que se conviertan en aprendices eficaces durante toda su vida y contribuyan a las sociedades de la información y el conocimiento (Catts \& Lau, 2009).

Por otra parte, la sociedad moderna exige que el modelo docente de trasmisión-recepción de la información (en el cual los estudiantes se comportan como receptores pasivos de información), cambie hacia un modelo que se corresponda con una cultura de aprendizaje cuyos fundamentos estén centrados en las nuevas formas de búsqueda, selección e interpretación de la información (Pozo, 1999). El principal reto por tanto está, en que el alumno sea capaz de asumir la responsabilidad de su propio aprendizaje y aprender a aprender a lo largo de la vida (Castilla, Chávez, Ramos, \& Bravo, 2014), por lo que es imprescindible que se comporten como gestores activos de información, utilizando de forma efectiva los recursos que ofrece la web.

Las cuestiones planteadas evidencian la importancia que tiene en el ámbito educativo, la actualización de profesores y estudiantes en lo referido a gestión de la información. Justamente, muchas instituciones y organismos internacionales, desde finales del pasado siglo y hasta los momentos actuales han desarrollado diversas de estrategias formativas para que las personas puedan acceder y usar eficientemente la información. Las estrategias se han desarrollado desde diversas perspectivas teóricas y usando diferentes vías, considerando además, las condiciones de los diferentes contextos (Alfonso, 2004; Antúnez, 2006; Antúnez, et al., 2012; Cordero, et al., 2009; Estrada, 2006; Espino, 2015; Fonseca, Lías, \& Gamboa, 2015; Gutiérrez, et al., 2009; Santovenia \& Linares, 2011; Hernández, 2010; Mainegra \& Miranda, 2012; Asencio \& Ibarra, 2015, 2016; Stuart, Delgado, Espín \& Céspedes, 2011).

Independientemente de lo logrado con la implementación de las estrategias mencionadas, existe un reconocimiento de que aún queda mucho por hacer para transformar la educación de manera adecuada para la preparación y la formación continua de las personas en la era de la información (Catts \& Lau, 2009), si se tiene en cuenta además que no siempre las instituciones cuentan con la infraestructura requerida para enfrentar esta tarea. Tal es el caso de la antigua Universidad Pedagógica "Félix Varela Morales" de la provincia de Villa Clara, Cuba, institución que presentaba serias limitaciones con los recursos informáticos y la conectividad a internet.

Sin embargo, en septiembre de 2015, derivado del proceso de integración de las instituciones de la Educación Superior en Cuba, la Universidad Pedagógica pasó a la estructura de la Universidad Central "Marta Abreu" de las Villas (UCLV), lo cual mejoró notablemente la infraestructura tecnológica y determinó la necesidad de implementar una estrategia para la actualización de la gestión de la información en estudiantes y profesores de la sede pedagógica. Precisamente este artículo se enmarca dentro de las acciones desplegadas en la estrategia y aborda la experiencia en la concepción e implementación de un programa curricular de carácter optativo dirigido a los estudiantes que cursan la Licenciatura en Educación en la sede pedagógica de la universidad mencionada.

\section{Método}

En este apartado se analizará el diseño metodológico que sustenta la experiencia en la concepción e implementación de una asignatura del currículo optativo en la formación de profesores de la 
sede pedagógica en la universidad de referencia, dirigida hacia la actualización de la gestión de la información para elevar la cultura informacional de los estudiantes. Además, en este epígrafe se presentará la caracterización del programa, así como la descripción de su implementación en la práctica.

\section{Diseño metodológico de la experiencia}

Es preciso señalar que el trabajo que se presenta se ubica en la categoría de estudio de caso y que, aunque se aplican métodos y procedimientos de investigación, no se alcanza el rigor de una investigación científica por lo que no se declara como tal. En general la metodología seguida se basó en la perspectiva Mixta (Sampiere, Collado \& Lucio, 2006), que facilita la combinación de un análisis cualitativo con un análisis cuantitativo.

Las primeras evidencias concretas que revelaron las necesidades de los estudiantes de la sede pedagógica fueron los bajos resultados alcanzados en la prueba de diagnóstico institucional, que sobre el tema de información científica y desarrollo de habilidades informacionales fue aplicada a los alumnos de tercer año de todas las carreras de la UCLV en febrero de 2016. Esta situación era de esperar, ya que la experiencia del trabajo diario de los autores de este trabajo en el área de la información científica, vislumbró que aún los estudiantes desconocían las posibilidades que le ofrecían las tecnologías e incluso los propios profesores manifestaban carencias en ese sentido. Derivado de lo anterior, fue considerado como problema el siguiente: ¿Cómo elevar la preparación de los estudiantes de las carreras de Licenciatura en Educación de la sede pedagógica de la UCLV para lograr la actualización en la información científica que requiere el desarrollo científico técnico actual?

Con vista a dar solución al problema planteado, se propone como objetivo: Diseñar e implementar un programa curricular de carácter optativo enfocado hacia la actualización de la gestión de la información para elevar la cultura informacional de los estudiantes de las carreras de Licenciatura en Educación de la sede pedagógica de la UCLV.

Entre los métodos empleados se destacaron: el analítico-sintético, el sistémicoestructural, la modelación, la observación, las entrevistas individuales, las entrevistas grupales, las encuestas, el análisis documental, el análisis de contenido y triangulación de los datos cualitativos obtenidos, el análisis estadístico descriptivo y la investigación-acción, entre otros. En especial, tuvo una significativa importancia la aplicación del método de investigación-acción, como herramienta indispensable para transformar entornos mediante un sistema ordenado de acciones que promueven el cambio, la mejora constante y la vinculación entre la teoría y la práctica (Cruz, 2015).

\section{Caracterización del programa optativo}

El programa optativo pretende ofrecer las bases teórico-prácticas relacionadas con la gestión de la información a fin de elevar la cultura informacional, la cual está ligada a la capacidad conceptual, procedimental y actitudinal para reconocer, desarrollar y potenciar el manejo integral de la información en el trabajo académico y científico que los estudiantes deben realizar en la carrera de Licenciatura en Educación. En este programa, el concepto de cultura informacional fue considerado como categoría rectora y eje central alrededor del cual fueron estructurados los diferentes temas, desde la perspectiva no solo de responder a las necesidades actuales de información determinadas por el desarrollo científico técnico, sino de trascender hacia el reconocimiento en los estudiantes de la necesidad de su actualización permanente a lo largo de toda la vida.

La cultura y la información en la sociedad moderna, son categorías que están íntimamente conectadas y guardan una relación de interdependencia recíproca (Menou, 2002). Así, la transferencia de información define las relaciones sociales que forman la estructura de la 
sociedad, contribuyendo a la evolución de la cultura y viceversa, la estructura social determina la cultura al proporcionar el marco en que se produce o se comunica (Ponjuan, 2002).

La propia autora mencionada, insiste en el concepto de cultura como un complejo de estrategias por medio de las cuales una sociedad intenta maximizar su adaptación al ambiente físico, y considera la cultura informacional, como un proceso continuo de aprendizaje que provoca una constante aproximación al cambio que puede provocar fuertes efectos que se concretan en la innovación, la creación intelectual, y en determinadas experiencias para el desarrollo que se generan especialmente en el mundo académico Ponjuan (2002).

Al respecto, Uribe (2013), plantea que la cultura informacional abarca las actuales transformaciones sociales, culturales y tecnológicas, que asume el conocimiento como un proceso de construcción colectiva reconociendo el espacio global de información y las interacciones sociales y que está asociada con el auto-aprendizaje y la actualización permanente.

Por otra parte, Cárdenas \& Jiménez (2007) definen la cultura informacional como la adquisición de un conjunto de habilidades y conocimientos para el correcto uso de la información y poder así desenvolverse satisfactoriamente en su participación y desempeño en la sociedad. Estas autoras enfatizan en que crear una cultura informacional no es fácil, que no es sencillo modificar las actitudes de los individuos, alterar su voluntad frente al desarrollo de la sociedad, porque conlleva una transformación profunda que impacta los valores, políticas, conductas y normas.

En esta misma línea de pensamiento, pero ahora desde la perspectiva didáctica, el empleo de la categoría cultura informacional, como concepto básico del programa referido, es que el mismo puede abarcar los diferentes componentes del contenido: conocimientos, habilidades y valores (Álvarez,1999), también reconocidos como contenidos conceptuales, procedimentales y actitudinales (Coll,1999; Coll \& Solé,1989; Pozo,1999; Nieda \& Macedo,1997), mientras que otros términos usados en la práctica internacional, tales como: alfabetización informacional, habilidades informacionales, competencias informacionales, gestión de la información, entre otros, solo se enmarcan en algunas aristas del contenido como componente del proceso de enseñanza-aprendizaje.

Hasta aquí, se ha ofrecido una breve argumentación de la categoría cultura informacional, para justificar la selección del concepto como categoría básica del programa. Seguidamente se ofrecerá una síntesis de los elementos fundamentales del programa de la asignatura en su versión final; es importante destacar que la concepción del programa se fue construyendo desde la práctica, a partir del empleo de métodos de investigación, en especial el método de investigaciónacción, por lo que estuvo sometido a ciclos de mejoramiento continuo durante el tiempo de desarrollo de la experiencia.

Título: La gestión de la información y la cultura informacional en la formación docente.

\section{Objetivos generales:}

1.- Explicar las bases conceptuales que sustentan la gestión de la información y la cultura informacional en las condiciones de la sociedad actual, para apoyar el trabajo académico y científico que requiere la carrera de Licenciatura en Educación.

2.- Desarrollar las habilidades informacionales para acceder, recuperar y evaluar la información relacionada con los temas de la especialidad, desde fuentes confiables atendiendo a sus necesidades, así como en su organización mediante el apoyo de Gestores de Referencias Bibliográficas, su procesamiento y comunicación de conocimientos a través de presentaciones orales y escritas. 
3.- Desarrollar valores y actitudes que respondan al reconocimiento del rol de la información científica en la sociedad actual y la necesidad de la elevación continua de la cultura informacional, sobre la base de los principios éticos y legales, así como la responsabilidad, honestidad, cooperación y autovaloración de sus progresos y desaciertos, para su perfeccionamiento personal y social.

Contenidos básicos de la asignatura:

Sistema de conocimientos:

La gestión de la información y la cultura informacional en la sociedad actual y en la Educación Superior cubana. Las fuentes de información. Fichado y normas bibliográficas. La evaluación crítica de las fuentes de información y principios éticos y legales. El impacto de las tecnologías de la información y las comunicaciones en el trabajo académico y científico. Las habilidades informacionales. Necesidades de información. Estrategias de búsqueda. Operadores booleanos. El Google Académico. Los recursos de información de la intranet de la UCLV. Bases de datos y revistas de la especialidad. La base EBSCO. La organización de la información. Gestores de Referencia Bibliográficos (GRB). El Gestor EndNote. El procesamiento de la información y la producción de conocimientos. La comunicación oral y escrita de conocimientos a través de presentaciones orales y escritas.

Sistema de habilidades:

1.- Elaborar fichas y referencias bibliográficas.

2.- Identificar las necesidades de información científico técnica.

3.- Formular estrategias de búsqueda utilizando los operadores booleanos.

4.- Buscar y recuperar información utilizando las herramientas de la web y los recursos de la intranet de la UCLV, así como en bases de datos y revistas de impacto internacional relacionadas con la especialidad.

5.- Organizar y evaluar críticamente la Información recuperada.

6.- Elaborar bibliotecas personales utilizando el gestor bibliográfico EndNote, de forma manual y exportando documentos de bases de datos, así como exportar referencias desde la biblioteca personal a documentos Word.

7.- Producir y comunicar la información a través de presentaciones orales y escritas.

Sistema de valores:

1.- Reconocer el rol de la gestión de la información científica en la sociedad actual y la necesidad de la elevación permanente de la cultura informacional.

2.- Desarrollar actitudes y valores que respondan a los principios éticos y legales relacionados con el uso de la información.

3.- Desarrollar la responsabilidad, honestidad, cooperación y autovaloración de sus progresos y desaciertos, para su perfeccionamiento personal y social.

Evaluación:

La forma de evaluación de la asignatura se estructura a partir de las evaluaciones frecuentes, de manera que pueda evaluarse el desempeño del alumno de forma directa y sistemática durante las actividades docentes, así como el cumplimiento de las tareas orientadas como trabajo independiente. 


\section{Implementación del programa optativo}

En este apartado se realizará una breve descripción de las actividades fundamentales realizadas durante la implementación del programa; al igual que en la concepción del programa la planificación de actividades, fueron ejecutadas durante la implementación del propio proceso.

La asignatura fue impartida durante en el curso 2017-2918 en los grupos de tercero y quinto de la carrera de Licenciatura en Educación en la especialidad de Pedagogía-Psicología, cuyas matrículas de estudiantes fueron de 18 y 19 respectivamente. Como parte del diagnóstico fue aplicada una encuesta escrita, así como entrevistas grupales en cada grupo.

Los instrumentos aplicados revelaron las siguientes regularidades:

- Desconocimiento de los términos relacionados con la información científica y las acciones generales de su tratamiento en el trabajo escolar.

- Limitaciones en el reconocimiento de las fuentes de información de uso tradicional en educación.

- Desconocimiento de los recursos de información que brinda la intranet de la UCLV.

- Bajo nivel en la búsqueda y recuperación de la información en la web.

- Desconocimiento del empleo de los GRB.

- Dificultades en la construcción de textos científicos y el empleo de citas y referencias bibliográficas.

En general, los resultados obtenidos en el diagnóstico permitieron precisar algunos detalles del programa preliminar elaborado, así como concretar las actividades que se implementarían en la práctica. Seguidamente se analizarán brevemente las actividades desarrolladas en cada uno de los temas que conforman el programa.

En el primer tema fueron tratadas las consideraciones generales acerca de la gestión de la información y la cultura informacional en la formación de profesionales de la educación y el mismo estuvo conformado por dos conferencias y dos clases prácticas. La primera conferencia se dedicó a la presentación de la asignatura y la aplicación de la encuesta y la entrevista grupal, mientras que en la segunda se analizaron los aspectos de orden teórico-conceptual relacionadas con la información científica, mediante el empleo de técnicas de trabajo grupal.

Las clases prácticas se dedicaron a la sistematización del trabajo con las fuentes impresas tradicionales, así como con el uso del correo electrónico y la navegación por internet en portales y sitios del área educativa. Como actividad final de tema se orientó una guía para el trabajo independiente, conformada por las tareas que se muestran seguidamente.

\section{TRABAJO INDEPENDIENTE. TEMA 1}

1.- Mencione ejemplos de cinco fuentes de información empleadas por usted en su trabajo académico y científico.

2.- Solicite dos libros y dos revistas relacionados con su especialidad en la Biblioteca de la Sede y elabore las fichas bibliográficas. En el caso de las revistas seleccione un artículo de cada una y elabore las fichas correspondientes

3.- Utilizando las fichas elaboradas escriba el formato de presentación de los libros y artículos de las revistas como bibliografías y/o referencias bibliográficas según las normas APA.

4.- Explique sus consideraciones sobre el impacto de las Tecnologías de la información y la Comunicación en el trabajo académico y científico en la etapa actual de desarrollo social. 
5.- Analice las necesidades de información que desde el punto de vista académico presenta en estos momentos. Identifique cinco términos (palabras clave o frases) derivadas de los conceptos en los que necesite buscar información en la web.

El tema 2 trató acerca de la búsqueda y recuperación de la información científica en la especialidad y fue conformado por una conferencia y tres clases prácticas. En la conferencia se profundizó en la elaboración de estrategias o perfiles de búsqueda utilizando los operadores lógicos y se ofreció una aproximación a las herramientas de búsqueda y recuperación de información en la web. En las clases prácticas se aplicaron los procedimientos para la recuperación de información en el Google Académico, los recursos de la intranet de la UCLV, la base EBSCO y las revistas científicas indexadas en bases de datos de impacto internacional en el área educativa. Como trabajo independiente de final del tema fueron orientadas las tareas que se presentan a continuación.

\section{TRABAJO INDEPENDIENTE. TEMA 2}

1.- Represente cinco estrategias o perfiles de búsqueda (enlazando términos con operadores lógicos) que respondan a sus necesidades de información en su tema particular de investigación. 2.- Escriba las referencias bibliográficas en el formato de la norma APA, de tres documentos relacionados con su tema de investigación, recuperados mediante el Google Académico.

3.- Desde el sitio de la UCLV recupere un documento vinculado con su tema de investigación en cada una de las fuentes siguientes: Repositorio Universitario, Biblioteca Digital "Félix Varela" y Revista Varela. Elabore las fichas bibliográficas de cada uno de los documentos descargados. 4.- Escriba las referencias bibliográficas en el formato de la norma APA, de tres documentos relacionados con su tema de investigación recuperados desde la Base de Datos EBSCO.

5.- Analice tres revistas científicas relacionadas con su tema de investigación y para cada una de ellas:

a) Mencione su nombre completo.

b) Explique su perfil.

c) Señale las Base de Datos en las que está indexada.

d) Escriba los datos de uno de los artículos que más le interesó.

En el tercer tema fue tratada la organización de la información mediante los GRB y se estructuró en una conferencia y dos clases prácticas. En la conferencia se destacó la importancia del empleo de los GRB, profundizándose en el gestor EndNote y en su uso para la elaboración de bibliotecas personales; por su parte, en las clases prácticas los estudiantes crearon sus bibliotecas personales, a partir de la entrada manual de datos y exportando desde bases de datos, así como ejercitaron la citación y elaboración de bibliografías desde un texto en Word. La guía orientada como trabajo independiente se muestra seguidamente.

TRABAJO INDEPENDIENTE. TEMA 3

1.- Caracterice la Biblioteca Personal que tiene elaborada hasta la fecha, mostrando los datos que se solicitan en la tabla siguiente:

\begin{tabular}{|l|l|l|l|l|}
\hline $\begin{array}{l}\text { Total de } \\
\text { registros }\end{array}$ & $\begin{array}{l}\text { Grupos de } \\
\text { documentos } \\
\text { creados }\end{array}$ & $\begin{array}{l}\text { Incorporados } \\
\text { de forma } \\
\text { manual }\end{array}$ & $\begin{array}{l}\text { Recuperados } \\
\text { del Google } \\
\text { Académico }\end{array}$ & $\begin{array}{l}\text { Recuperados } \\
\text { de EBSCO }\end{array}$ \\
\hline & & & & \\
\hline
\end{tabular}

2.- Atendiendo al conjunto documental que compone la Biblioteca Personal que tiene elaborada hasta la fecha, escriba la cantidad, actualidad y tipos de documentos según los datos que muestra la tabla que aparece seguidamente. 


\begin{tabular}{|l|l|l|l|l|}
\hline Tipo de fuente & 2015-2011 & 2010-2006 & 2005-2001 & al 2001 \\
\hline Nivel de actualidad & & & & \\
\hline Libros & & & & \\
\hline Artículos impresos & & & & \\
\hline Artículos on line & & & & \\
\hline Textos en idioma inglés & & & & \\
\hline Otras & & & & \\
\hline
\end{tabular}

3.- Muestre la página inicial de su Biblioteca Personal mediante una captura de pantalla e insertada en el documento Word donde deben aparecer las respuestas a las tareas.

4.- Exprese sus consideraciones acerca de la significación que para usted tiene el empleo de los gestores de referencias bibliográficas en el trabajo académico y científico, para su actividad profesional presente y futura.

5.- Elabore un texto relacionado con el tema de su investigación, donde enlace de forma coherente algunas referencias tomadas del conjunto documental de su biblioteca personal con la bibliografía correspondiente, utilizando los recursos con que cuenta el gestor EndNote. La extensión del texto incluyendo la bibliografía debe tener como mínimo dos cuartillas.

Por último, el tema 4 fue dedicado a la producción y comunicación de la información en la especialidad que fue conformado en una conferencia, una clase práctica y un seminario final que resumía todos los contenidos abordados en la asignatura. La conferencia abordó los aspectos generales sobre el procesamiento de la información y la producción de conocimientos, profundizándose en las características fundamentales del texto científico y sus diferentes formas de presentación y comunicación. La clase práctica sirvió para ejercitar la construcción de textos y el empleo de la norma APA en citas y referencias.

El seminario final estuvo enfocado a la sistematización de los contenidos de la asignatura mediante la presentación de la biblioteca personal elaborada y la demostración de su empleo en la construcción de textos científicos; seguidamente se muestra la guía correspondiente.

\section{GUÍA PARA EL SEMINARIO FINAL}

\section{PRESENTACIÓN DE LOS RESULTADOS OBTENIDOS EN LA ASIGNATURA.}

1.- Mostar el conjunto documental registrado en la biblioteca y caracterizar los mismos atendiendo a:

a) Total de registros y nivel de actualización de documentos.

b) Nivel de satisfacción de las necesidades de información.

c) Estrategias de búsqueda empleadas.

d) Fuentes de información de dónde fueron recuperados los documentos. 
2.- Explicar (tomando como ejemplo uno de los registros de la biblioteca) los campos de datos llenados, a fin de obtener la referencia bibliográfica atendiendo al tipo de documento.

3.- Analizar la vista previa que muestra el sistema (en el formato APA 6ta ed.) tomando como ejemplo los siguientes tipos de documentos:

a) Libro.

b) Artículo electrónico.

4.- Demostrar cómo incorporar una referencia de su biblioteca personal en un texto científico relacionado con su tema de investigación.

\section{Resultados y discusión}

En este apartado se analizarán los resultados cuantitativos y cualitativos obtenidos con la aplicación del programa.

Desde el punto de vista cuantitativo, para valorar los resultados, fueron seleccionados cuatro indicadores, que fueron evaluados al final del semestre, teniendo en cuenta los datos recogidos durante todo el proceso, a partir de la aplicación de diferentes métodos, tales como: la observación, el análisis de documentos, la aplicación de cuestionarios y encuestas, entrevistas, guías de autovaloración, entre otros. Entre los indicadores fueron considerados los siguientes:

I.- Dominio de conocimientos y habilidades.

II.- Satisfacción por las actividades realizadas.

III.- Motivación por continuar elevando su cultura informacional.

IV.-Valoración de la importancia de los temas tratados.

Los indicadores mencionados fueron medidos en una escala ordinal, que consideraba tres niveles: Alto (A), Medio (M) y Bajo (B).

En el indicador I, el 60\% de los estudiantes fue evaluado en el nivel alto, mientras que el $32 \%$ y el $8 \%$ correspondieron a los niveles medio y bajo respectivamente, lo cual se corresponde con la figura 1.

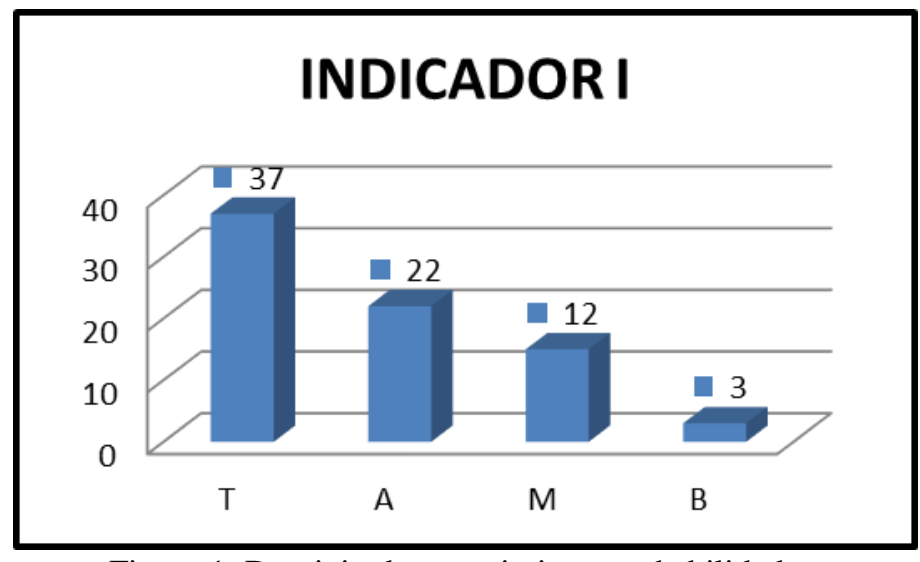

Figura 1: Dominio de conocimientos y habilidades.

Con relación al indicador II, en el nivel alto fueron ubicados el $86 \%$ de los estudiantes, en el medio el $8 \%$ y en el bajo el 6\%. Lo anterior se puede comprobar en la figura 2. 


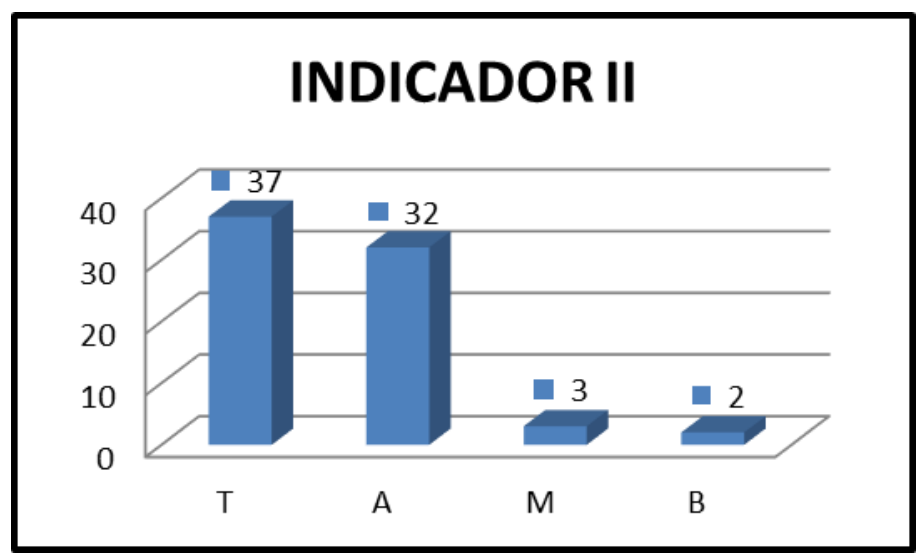

Figura 2: Satisfacción por las actividades realizadas

Derivado de la evaluación del indicador III, fueron considerados, al 78\% de los alumnos en el nivel alto, el $16 \%$ en el medio y el $6 \%$ en el bajo, lo que se corresponde con el gráfico de la figura 3 .

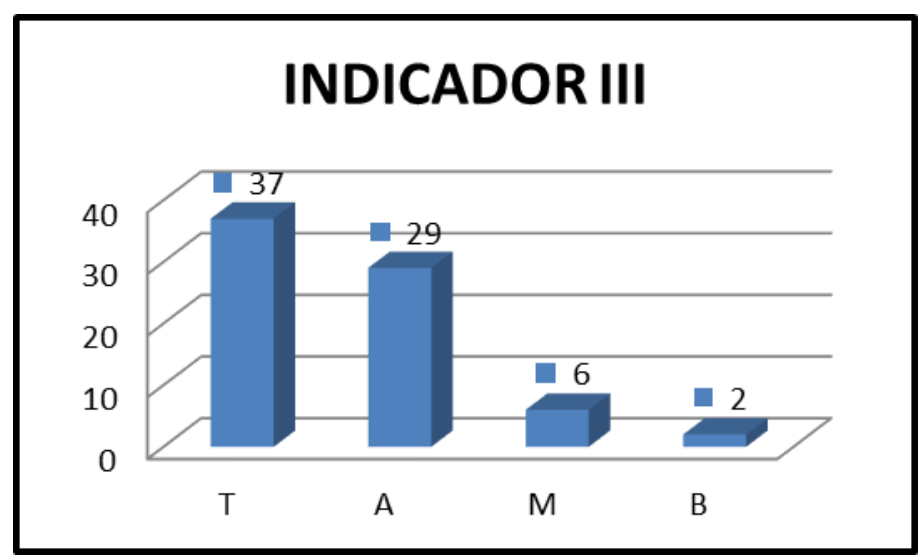

Figura 3: Motivación por continuar elevando su cultura informacional.

Por último, en el indicador IV, el 75\% de los alumnos fueron evaluados en el nivel alto, mientras que el $22 \%$ y el $3 \%$ estuvieron en los niveles medio y bajo respectivamente; lo anterior se puede ratificar en desde el análisis de la figura 4 , que se muestra seguidamente.

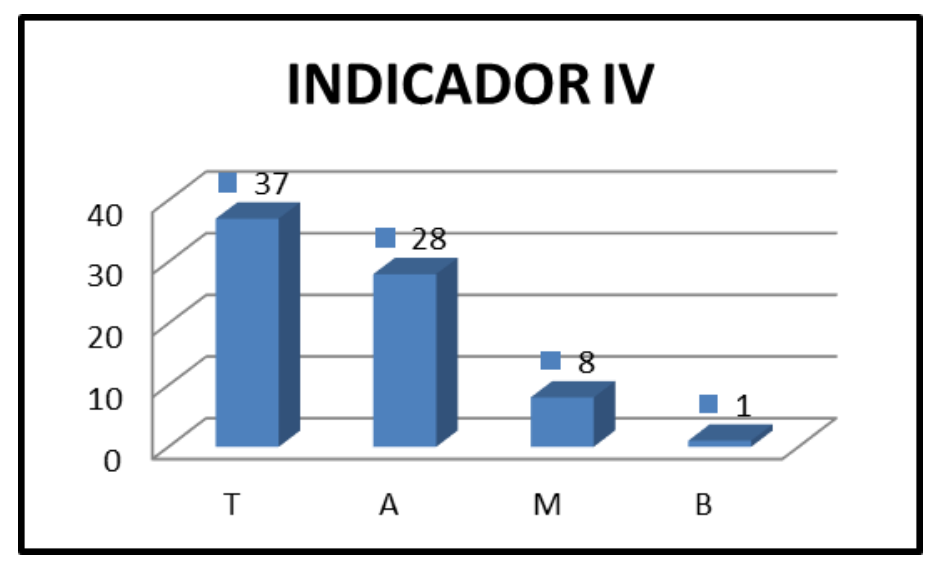

Figura 4: Valoración de la importancia de los temas tratados. 
Puede resultar de interés, también, destacar como parte de los resultados alcanzados en la implementación de la propuesta, analizar las notas obtenidas por los estudiantes de ambos grupos al final de semestre; la tabla 1 y la figura 5 muestran los datos correspondientes.

Tabla 1.

Notas obtenidas en la asignatura

\begin{tabular}{ccccc}
\hline Año & Total & Excelente & Bien & Aprobado \\
\hline 3ro & 18 & 1 & 11 & 6 \\
\hline 5to & 19 & 5 & 6 & 8 \\
\hline Total & 37 & 6 & 17 & 14 \\
\hline
\end{tabular}

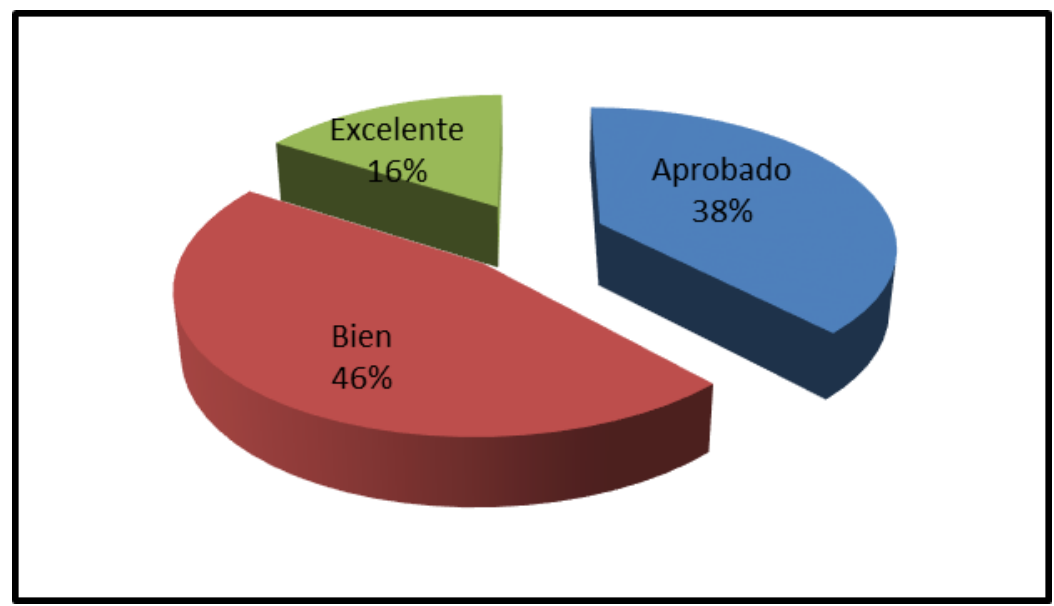

Figura 5: Notas obtenidas en la asignatura.

Por último, se hará referencia a los datos cualitativos obtenidos en la experiencia. No es posible en el marco de este artículo, por limitaciones de espacio, ofrecer toda la riqueza de los datos recogidos, los cuales aportaron los criterios y opiniones vertidas por los estudiantes a partir de los instrumentos aplicados y las autovaloraciones realizadas. Solo se mostrarán algunos ejemplos, que se presentan a continuación:

"Los contenidos impartidos nos dieron herramientas para desarrollar las habilidades informacionales".

"Nos prepara para lograr ser ese profesional competente que demanda la sociedad".

"Los contenidos recibidos fueron de gran interés y han sido profundamente útiles para gestionar información confiable".

"Nos enseñó a indagar en fuentes de información que antes no conocíamos".

"Mi motivación por continuar elevando mi cultura informacional es alta".

"Los conocimientos adquiridos me ayudarán en los trabajos de curso y diploma".

"Creo que la biblioteca personal es uno de los recursos que me va a ayudar más en mi trabajo futuro".

"Considero que todo profesional de la educación tiene que elevar constantemente su cultura informacional".

"Me siento muy motivada para seguir satisfaciendo mis necesidades de información".

"Estoy muy satisfecho y creo que el curso optativo tiene gran importancia".

"Me gustó mucho la navegación por las bases de datos y las revistas": 
"Debo profundizar en la organización de los datos y en el empleo de la norma APA".

"Todavía tengo que seguir practicando en exportar referencias desde bases de datos".

Es importante destacar que entre las opiniones de los estudiantes de quinto año (último año de la carrera) fue recurrente el criterio de que la asignatura debió ser impartida en años anteriores, pues de esta forma podían utilizar los conocimientos adquiridos en sus trabajos de curso y diploma.

\section{Conclusion}

En este trabajo se ha presentado la experiencia en la concepción e implementación de un programa curricular de carácter optativo dirigido a la elevación de la gestión de la información y la cultura informacional de los estudiantes en formación docente.

La concepción del programa se centró en el concepto de cultura informacional, que fue considerado como categoría rectora, desde la perspectiva no solo de responder a las necesidades actuales de información determinadas por el desarrollo científico técnico, sino de trascender hacia el reconocimiento en los estudiantes de la necesidad de su actualización permanente a lo largo de toda la vida.

respecto a los resultados obtenidos, la medición de los indicadores y el análisis de los datos cualitativos recogidos durante la experiencia, permitieron comprobar que los estudiantes elevaron su nivel de dominio en los conocimientos, habilidades y valores relacionados con los contenidos abordados, lo que demuestra la influencia positiva del programa en el contexto particular en donde fue aplicado.

En general, se considera como una de las cuestiones más novedosas del programa implementado, que enfoca de manera intencional, el desarrollo de la motivación de los estudiantes por continuar profundizando en el tema de la cultura informacional para responder a las demandas el desarrollo científico técnico, lo cual contribuye a que reconozcan y actúen consecuentemente con los cambios informacionales, que como parte de la revolución cultural en la que está inmersa la sociedad actual, afecta todas las esferas de la vida de los seres humanos.

\section{Referencias}

Aleixandre, R., Alcaide, G., González, J., \& Alonso, A. (2011). Fuentes de información bibliográfica (I). Fundamentos para la realización de búsquedas bibliográficas. Acta Pediátrica Española, 69(3), 131-136. RESULTADOS

Antúnez, et al., (2012). Curso virtual de redacción científica e infotecnología sobre la plataforma MOODLE: resultados y experiencias. Píxel-Bit. Revista de Medios y Educación, 41, 173183.

Antúnez, G. (2006). Curso online Redacción Científica y las Tecnologías de la Información y las Comunicaciones. Revista Electrónica de Veterinaria REDVET, VII(8). En: http://www.veterinaria.org/revistas/redvet/n080806.html

Alfonso, J. E. (2004). Curso a distancia: Cómo preparar y publicar artículos científicos. En: http://files.sld.cu/redenfermeria/files/2011/05/tesis-final-1-03.pdf

Álvarez, C. (1999). Didáctica. La escuela en la vida. Tercera Edición, La Habana: Editorial Pueblo y Educación.

Asencio, E. \& Ibarra, N. (2015). Plan de acción para promover la participación de docentes en redes y publicaciones en revistas educativas. Revista Educare 19(3), 24-38.

Asencio, E. \& Ibarra, N. (2016). Superación profesional para promover las publicaciones en revistas científicas. Revista Universidad y Sociedad, 8(2),79-88. 
Cárdenas, A. \& Jiménez, N. Acceso universal a la información: globalización, cultura y alfabetización. Acimed 15(1)

Castilla, L., Chávez, M., Ramos, J., \& Bravo, T. (2014). Alternativa orientadora en alfabetización informacional para estudiantes universitarios desde la biblioteca. Informacion, Cultura y Sociedad, (30), 105-126.

Catts, R., \& Lau, J. (2009). Hacia unos indicadores de Alfabetización Informacional. En: http://www.peri.net.ni/pdf/docALFIN2014/seminario.pdf

Coll, C. (1999). Psicología de la instrucción: la enseñanza y el aprendizaje en la Educación Secundaria. España: Editorial Horsori.

Coll, C. \& Sollé, I. (1989). Aprendizaje significativo y ayuda pedagógica. España: Editorial Horsori.

Cordero, G. et al., (2009). Publicación científica y evaluación docente en México. Tiempo de Educar, 10(19), 149-168.

Cruz, K. A. (2015). La formación inicial de investigadores. Raximhai, 11(4), 91-100.

Estrada, A. (2006). Curso Redacción científica y publicaciones académicas. Facultad de Letras y Ciencias Humanas, Madrid. Universidad Nacional Mayor de San Marcos. En: http://redaccioncientifica.blogspot.com/

Espino, S. (2015). La enseñanza de estrategias de escritura y comunicación de textos científicos y académicos a estudiantes de posgrado. Revista Mexicana de Investigación Educativa, 20(66), 959-976.

Fonseca, C., Lías, Y. M. \& Gamboa, R. (2015). Autores e información del artículo. Revista Atlante: Cuadernos de Educación y Desarrollo. Recuperado de http://www.eumed.net/rev/atlante/2015/05/talleres.html

Gutiérrez, et al., (2009). Curso a distancia para la redacción de artículos científicos. Medisur, 7(2). $12-20$.

Hernández, A. (2010). Saberes, habilidades y estrategias utilizadas en la escritura de un texto científico: ensayo o artículo. Matices. Revista de posgrado, 5(13), 245-249.

Mainegra, D., \& J. Miranda (2012). Una propuesta para mejorar la comunicación de los resultados de la investigación educativa de la UCP "Rafael María de Mendive. Revista Mendive, 11(41).

Menou, M. (2002). Information Literacy in National Information and Communications Technology (ICT) policies: The Missed Dimension, Information Culture.

Nieda, J. \& Macedo, B. (1997). Un currículo científico para estudiantes de 11 a 14 años. España: Editado por: OIE-UNESCO.

Pinto, M., Sales, D., \& Martínez-Osorio, P. (2009). El personal de la biblioteca universitaria y la alfabetización informacional: de la autopercepción a las realidades y retos formativos. Revista Española de Documentación Científica, 32(1), 60-80,

Pozo, J. (1999). Aprendizaje de contenidos y desarrollo de capacidades en la Educación Secundaria. En Psicología de la instrucción. España: Editorial Harson.

Ponjuan, G. (2002). Papel de la colaboración entre líderes de varios sectores para la creación de una Cultura informacional.

Sampieri, R. H., Collado, C. F., \& Lucio, P. B. (2006) Metodología de la investigación. Cuarta edición. México: Interamericana (Ed.).

Santovenia, J. R., \& Linares, M. P. (2011). Proponen curso de redacción de documentos científicos desde la perspectiva de las Ciencias de la información. Ciencias de la Información, 42(2), 71-73.

Stuart, M., Delgado, M., Espín, R., \& Céspedes, Z. (2011). Búsqueda y recuperación de información en Internet desde una perspectiva de los usuarios en la educación superior. Pedagogía Universitaria, 16(1), 70-87.

Uribe, A. (2013). El Programa de ALFIN del Sistema de Bibliotecas de la Universidad de Antioquia ahora es: "Cultura Informacional". Recuperado de http://alfincolombia.blogspot.com/2013/01/otras-publicaciones-recientes.html

Varela, C. (2007). Blended learning para un programa de desarrollo de habilidades informacionales en la Biblioteca de la Escuela Universitaria de Formación de Profesorado de Lugo. Ibersid, 275-283. 
Winston, J., Barbosa, J., Marciales, G., \& Castañeda, H. (2010). Reconceptualización sobre competencias informacionales. Una experiencia en la Educación Superior. Revista de Estudios Sociales, 37, 121-142. 